\title{
Evaluation of seminal oxidation-reduction potential in male infertility
}

\author{
Manesh Kumar Panner Selvam ${ }^{1}$ (D) | Renata Finelli ${ }^{1}$ (D) | Ashok Agarwal $^{1}$ (D) | \\ Ralf Henkel ${ }^{1,2}$ (i)
}

\author{
${ }^{1}$ American Center for Reproductive \\ Medicine, Cleveland Clinic, Cleveland, $\mathrm{OH}$ \\ USA \\ ${ }^{2}$ Department of Medical Bioscience, \\ University of the Western Cape, Bellville, \\ South Africa \\ Correspondence \\ Ashok Agarwal, American Center for \\ Reproductive Medicine, Cleveland Clinic, \\ Mail Code X-11, 10681 Carnegie Avenue, \\ Cleveland, OH 44195, USA. \\ Email: agarwaa@ccf.org
}

\begin{abstract}
The role of oxidative stress in male infertility has been broadly recognised, and the search for a new marker to determine the redox environment in semen has gained considerable interest. Oxidation-reduction potential (ORP) or redox potential, is a measure of the electron transfer from antioxidants to oxidants and provides information on the redox balance. In this review, the benefits of ORP as a new oxidative stress marker, the protocol for its evaluation and the importance of its measurement in the context of male infertility are discussed. In association with the standard semen analysis, seminal ORP has been analysed to evaluate semen quality and male fertility status. However, further studies are required to establish its use in assisted reproductive techniques (ART) practice.
\end{abstract}

KEYWORDS

male infertility, oxidation-reduction potential, oxidative stress

\section{1 | BACKGROUND}

Oxidation-reduction potential (ORP) or redox potential is a measure of the tendency of a molecule to lose or gain electrons. The ORP levels of a system determine the transfer of electrons from lower ORP-valued molecules (reductants) to higher ORP-valued molecules (oxidants). Currently, ORP testing is widely used in the evaluation of the global redox status in human biological fluids, such as urine (Cao, He, Bai, \& Liu, 2016) and plasma (Polson, Villalba, \& Freeman, 2018). In the case of traumatic brain injury, ORP is used as a marker to assess the severity of the injury (Bjugstad et al., 2016). The ORP measurement has a higher power in predicting severity and survival outcomes than other tools usually used in this pathological condition, such as the Injury Severity Score, Abbreviated Injury Scale and Glasgow Coma Scale (Bjugstad et al., 2016). Cao et al. (2016) reported higher ORP levels in urine samples of diabetic patients compared to normal healthy men. In athletes, ORP levels were evaluated in blood plasma to assess their redox status (Stagos et al., 2015). Further, Polson et al. (2018) optimised the procedure for measuring ORP levels in plasma collected from heparinised blood samples (Polson et al., 2018). Recently, the evaluation of ORP has been introduced to measure oxidative stress in seminal fluid of men with infertility (Agarwal, Sharma, Roychoudhury, Du Plessis, \& Sabanegh, 2016).

Oxidative stress has been correlated with high levels of reactive oxygen species (ROS) and poor sperm quality in infertile men (Agarwal, Rana, et al., 2018; Gharagozloo \& Aitken, 2011; Saleh \& Agarwal, 2002). An estimate of $30 \%-80 \%$ of infertile men are diagnosed with seminal oxidative stress, accounting for more than 55 million people worldwide (Agarwal, Parekh, et al., 2019). The condition of these infertile men having poor semen quality and high levels of oxidative stress has been described as MOSI (Male Oxidative Stress Infertility; Agarwal, Parekh, et al., 2019). At physiological concentrations, ROS are intimately involved in the regulation of several sperm functions such as capacitation, acrosome reaction, hyperactivation and membrane fusion between spermatozoa and oocyte (Aitken, 2017; Du Plessis, Agarwal, Halabi, \& Tvrda, 2015). However, ROS can lead to cellular damage and infertility if the concentration overwhelms the seminal antioxidants. Sperm motility is mainly affected by oxidative stress as mitochondrial activity and 
ATP generation is affected by the increased levels of ROS (Wang, Sharma, Gupta, et al., 2003). Mitochondrial membrane potential (MMP) is a direct marker of the cellular energy status and mitochondrial functionality (Marchetti, 2002). It negatively correlates with the ROS levels and is affected by oxidative stress (Wang, Sharma, Gupta, et al., 2003). Further, ROS has a significant negative impact on sperm morphology by altering the acrosome structure. El-Taieb, Ali, and Nada (2015) reported an increased number of acrosome abnormalities (e.g. acrosome swelling and inclusions) in patients with high levels of malondialdehyde, a well-known marker of lipid peroxidation (El-Taieb et al., 2015). Seminal oxidative stress has also been associated with the induction of cellular apoptosis, resulting in a reduced number of viable spermatozoa and increased DNA damage (Mahfouz et al., 2010; Wang, Sharma, Sikka, et al., 2003). A high rate of sperm DNA damage can trigger the apoptotic process, as spermatozoa lack base repair mechanisms (Aitken, De luliis, Gibb, \& Baker, 2012). Hence, the evaluation of oxidative stress provides an insight into the semen quality and male fertility potential.

Currently, several methods are used for the laboratory evaluation of oxidative stress in semen samples (Agarwal, Qiu, \& Sharma, 2018); these are categorised as direct and indirect tests (Table 1). The direct tests measure the levels of ROS in semen samples, whereas the indirect tests evaluate the amount of individual or global antioxidants or oxidised products such as malondialdehyde and the modified base 8-hydroxy-2'-deoxyguanosine (8-OHdG). The chemiluminescence assay is one of the widely used direct tests to measure ROS levels using a luminol probe that emits photons (Agarwal, Ahmad, \& Sharma, 2015; Khan et al., 2014). Luminol can detect both intra- and extracellular ROS, but cannot discriminate between the different types of ROS present in semen samples. Furthermore, it is a time-consuming technique and requires a minimum of $800 \mu \mathrm{l}$ of semen samples. Frozen semen, azoospermic or hyperviscous samples are not suitable for chemiluminescence assays, thus limiting their diagnostic potential (Aitken, Baker, \& O'Bryan, 2004). Redox homeostasis is established by the fine balance between the oxidants and reductants. Hence, measurement of only either oxidants or reductants does not provide enough evidence to support the diagnosis of oxidative stress. There is a need to identify a new marker of oxidative stress to fully determine the redox potential status in semen. The ORP testing was introduced to measure the global balance of the redox potential in semen samples (Agarwal, Roychoudhury, Sharma, et al., 2016). A strong correlation has been observed between seminal ORP and various sperm parameters such as concentration, motility and morphology, indicating ORP as a marker of seminal oxidative stress (Agarwal, Panner Selvam, et al., 2019). Furthermore, ORP has been shown to be a feasible adjunct test to conventional semen analysis (Agarwal, Panner Selvam, et al., 2019; Agarwal, Sharma, et al., 2016; Agarwal \& Wang, 2017; Arafa et al., 2018; Douglas, Parekh, Kahn, Henkel, \& Agarwal, 2019).

\section{2 | TECHNOLOGY AVAILABLE TO MEASURE ORP}

\section{1 | MiOXSYS analyser}

The analysis of oxidative stress is carried out in conjunction with the standard semen analysis to identify the aetiology associated with poor semen quality and male infertility. The MiOXSYS analyser (Male Infertility Oxidative System; Aytu BioScience; Figure 1a) is an ultrahigh impedance electrometer, which evaluates the ORP levels in semen sample by measuring the electron transfer from the antioxidants to the oxidants (Agarwal, Sharma, et al., 2016). Unlike other techniques, ORP measurement by MiOXSYS is an easily performed technique that does not require a specific personnel training or sample processing. Moreover, it analyses ORP in a small amount of both fresh or frozen samples $(30 \mu \mathrm{l})$ in $<4 \mathrm{~min}$, with stable results for up to 120 min after collection of the semen samples (Agarwal, Sharma, et al., 2016). Hence, it is recommended to freeze the sample if it cannot be evaluated within 120 min after liquefaction.

\section{2 | Other probes and analysers}

The ORP sensors work similarly to the $\mathrm{pH}$ sensors. They detect the ORP of the solution by measuring the difference in redox potential between a working electrode and a reference electrode made up of gold or platinum. The manufacturer Sensorex Inc developed several types of sensors, depending on its application and the resistance to increasing temperature (up to $100^{\circ} \mathrm{C}$; https://senso rex.com/). ORP sensors can be wirelessly connected to an android tablet or phone (Camlab) to allow the storage and retrieval of previous results (https://www.camlab.co.uk/). Other sensors can simultaneously detect ORP, temperature and $\mathrm{pH}$ as well as other water

\begin{tabular}{|c|c|}
\hline Direct tests & Indirect tests \\
\hline Chemiluminescence & Myeloperoxidase or Endtz test \\
\hline Nitro blue tetrazolium (NBT) & Lipid peroxidation-malondialdehyde levels \\
\hline Cytochrome $\mathrm{c}$ reduction test & $\begin{array}{l}\text { Evaluation of inflammatory mediators (chemokines } \\
\text { and interleukins) }\end{array}$ \\
\hline $\begin{array}{l}\text { Fluorescein isothiocyanate (FITC)- } \\
\text { labelled lectins }\end{array}$ & $\begin{array}{l}\text { Antioxidants, micronutrients, vitamins (vitamin E and } \\
\text { vitamin C) }\end{array}$ \\
\hline Electron spin resonance (ESR) & Total antioxidant capacity (TAC) \\
\hline Oxidation-reduction potential (ORP) & DNA damage-8-hydroxy-2'-deoxyguanosine levels \\
\hline
\end{tabular}

TABLE 1 Most commonly used tests to evaluate oxidative stress in the seminal plasma 

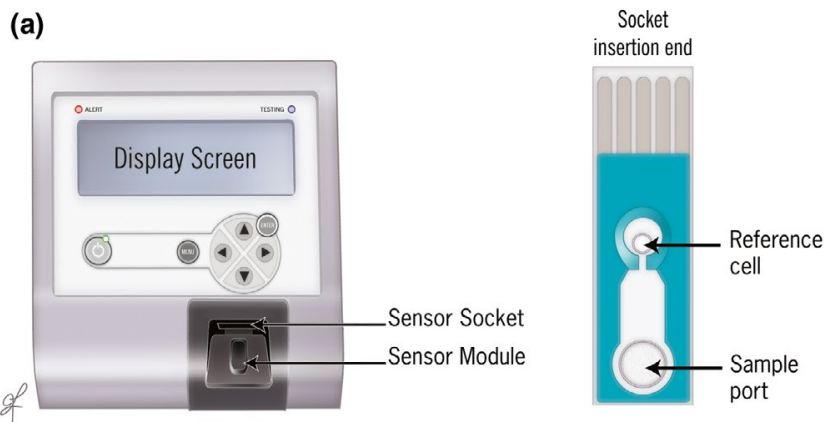

(b)

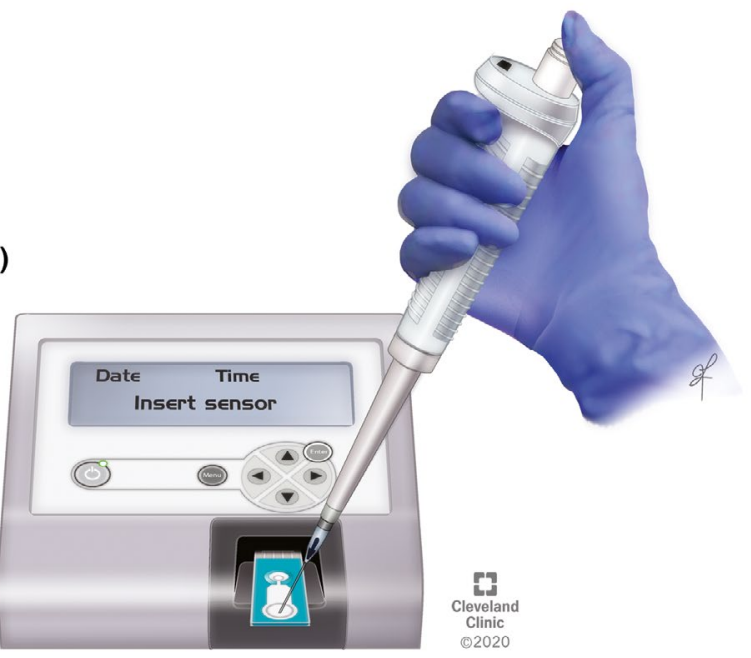

FIGURE 1 (a) MiOXSYS analyser and sensor; (b) Loading of semen sample into the application port, filling the reference electrode

characteristics such as dissolved oxygen, conductivity, salinity, ammonium, nitrate and chloride (Xylem Inc, https://www.xylem.com/ en-us/). In the medical field, the type of analyser varies in relation to the sample that is analysed, with the electrodes being smaller when the sample volume to be analysed is less. Pluschkell and Flickinger measured ORP in cell cultures using a Thermo Scientific Orion analyser (Pluschkell \& Flickinger, 1995), while the RedoxSYS Diagnostic System (Luoxis Diagnostics, which then merged into Aytu BioScience), the MiOXSYS precursor, was used to measure ORP in human blood plasma (Bjugstad et al., 2016; Polson et al., 2018; Stagos et al., 2015).

\section{3 | MEASUREMENT OF ORP USING MIOXSYS}

\section{1 | Principle}

The electrons transferred from the antioxidants (electron donators) to oxidants (electron acceptors) are measured by the MiOXSYS anaIyser based on the Nernst equation:

$$
E_{(\mathrm{ORP})}=E^{\circ}-R T / n F \ln ([\mathrm{Red}] /[\mathrm{Ox}])
$$

where $E$ = standard reduction potential; $R=$ the universal gas constant; $T=$ the absolute temperature; $n=$ number of moles of exchanged electrons; $F=$ Faraday constant; Red = concentration of reduced species; and $\mathrm{Ox}=$ concentration of oxidised species.

\section{2 | Sample collection and preparation}

\subsection{1 | Fresh or neat semen}

Samples collected after a sexual abstinence of 2-7 days are kept in the incubator at $37^{\circ} \mathrm{C}$ for $20 \mathrm{~min}$. After complete liquefaction, semen analysis is carried out to assess both macroscopic characteristics such as $\mathrm{pH}$, volume and colour, and microscopic characteristics such as concentration, motility and morphology (WHO, 2010). A small aliquot of semen sample $(30 \mu \mathrm{l})$ is analysed using the MiOXSYS.

\subsection{2 | Frozen semen}

The ORP measurement can be performed using frozen semen samples (Agarwal, Sharma, et al., 2018). Sample freezing does not influence the ORP values. Initially, semen samples should be thawed at $37^{\circ} \mathrm{C}$ before testing and vortexed to ensure proper mixing of the sample. Then, a small aliquot of sample $(30 \mu \mathrm{l})$ is used for ORP measurement.

\section{3 | Instrumentation and analysis}

The MiOXSYS sensor contains three electrodes: $\mathrm{Ag} / \mathrm{AgCl}$ reference electrode and two working electrodes made of platinum. A small amount of sample $(30 \mu \mathrm{l})$ is loaded on the sensor application port (Figure 1b). The sample flows through the sensor and fills the reference cell. It is very important to fully cover the application port to avoid the formation of any air bubbles. Once the sensor is introduced into the analyser, the electrochemical circuit is closed, and the measurement starts. The MiOXSYS analyser detects the voltage between the reference and the working electrodes every $0.5 \mathrm{~s}$ interval, and the final ORP is calculated after $\sim 120 \mathrm{~s}$ as the average of the last $10 \mathrm{~s}$ (or the last 20 readings) of the run. Results are expressed in millivolt ( $\mathrm{mV}$ ) as static ORP (sORP).

\subsection{Calculation and interpretation of results}

The ORP is influenced by the concentration of cells, and it is inversely proportional to the viability of cells (Pluschkell \& Flickinger, 1995). Therefore, the ORP must be normalised against the sperm concentration to obtain a value, which is representative of the semen. ORP levels are influenced by both the quantity (concentration) and quality (morphology) of the cells (Pluschkell \& 
Seminal Analysis

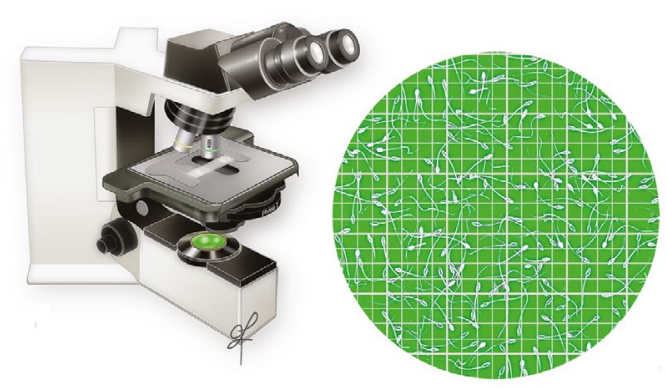

Sperm concentration $50 \times 10^{6} \mathrm{sperm} / \mathrm{mL}$
ORP Measurement

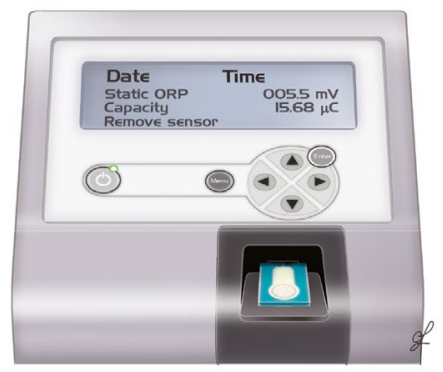

FIGURE 2 ORP value displayed on the analyser is normalised with the sperm concentration. Results are expressed as $\mathrm{mV} / 10^{6}$ spermatozoa/ml

\section{Normalized ORP $=\mathbf{5 . 5 / 5 0}=0.11 \mathrm{mV} / 10^{6} \mathrm{sperm} / \mathrm{ml}$}

Static ORP: $5.5 \mathrm{mV}$

Flickinger, 1995). In semen sample, the presence of immature or abnormal spermatozoa can reduce the availability of antioxidants as well as increase the generation of ROS (Agarwal, Hamada, \& Esteves, 2012). Hence, the normalisation process for ORP is crucial to distinguish the oxidative status between samples with the same concentration but with varying sperm quality. The normalised ORP is expressed as $\mathrm{mV} / 10^{6}$ spermatozoa/ml (Figure 2).

Seminal parameters can influence the efficiency of MiOXSYS to detect ORP. For instance, a sample characterised by poor liquefaction or high viscosity shows resistance in flow from the application port to the reference cell, making the analysis challenging. Moreover, the necessity to normalise the ORP with sperm concentration limits its use with azoospermic samples. In addition, temperature variation may influence the analysis, as the optimal working temperature is between 5 and $45^{\circ} \mathrm{C}$. Hence, it is important to thaw the frozen semen samples to $37^{\circ} \mathrm{C}$ before testing for ORP using MiOXSYS.

\subsection{Quality control}

Quality control (QC) is an essential step carried out by each laboratory to ensure both precision and accuracy of the results. The WHO guidelines strongly recommend the necessity of performing QC in andrology practice to guarantee the robustness of the analysis and minimise false results (WHO, 2010). The QC for the MiOXSYS analyser is carried out using two aqueous solution matrices provided by the manufacturer having known low (33-70 mV) and high (91-117 mV) ORP values. The QC checks of the instrument are performed prior to the testing of semen sample to ensure the device is functioning optimally. QC should also be done routinely to verify each new lot of sensors. Calibration of the instrument should be done before analysing the sample using a calibration verification key (CVK) provided by the manufacturer. Both sides of the CVK (side A: 99-101 mV and side B: 295.8-304.2 mV) are analysed, and the values must fall between the acceptance limits; otherwise, maintenance should be requested.

\section{4 | CLINICAL RELEVANCE OF ORP MEASUREMENT}

\section{1 | ORP and male infertility}

In a clinical andrology setting, ORP has been utilised as a reliable marker of oxidative stress in human semen samples (Agarwal, Roychoudhury, Bjugstad, \& Cho, 2016). Several studies have demonstrated the clinical relevance of ORP testing in the evaluation of male infertility (Agarwal et al., 2017; Agarwal, Henkel, Sharma, Tadros, \& Sabanegh, 2018; Agarwal, Sharma, et al., 2016; Agarwal \& Wang, 2017). In a preliminary study, Agarwal et al. reported a negative correlation between semen parameters, such as concentration and motility, and ORP levels in semen and in seminal plasma. Further reports have confirmed the negative association between sperm concentration, motility, total motile sperm count and morphology with seminal ORP levels (Agarwal et al., 2017; Agarwal, Henkel, et al., 2018; Agarwal, Roychoudhury, Sharma, et al., 2016; Agarwal, Sharma, et al., 2016; Agarwal \& Wang, 2017; Majzoub et al., 2018, 2019). In comparison with a chemiluminescence assay that measures ROS levels, seminal ORP shows a stronger negative correlation with sperm motility and morphology, suggesting ORP as a more reliable marker of oxidative stress than ROS in a clinical setting (Homa et al., 2019). However, although leucocytes are the main source of ROS production (Henkel, 2011), no correlation has been reported between seminal ORP and leucocyte concentration in semen samples (data from our laboratory).

Studies conducted to predict the ability of ORP in discriminating semen samples in men with abnormal parameters based on their fertility status from normal or fertile samples, respectively, are shown in Table 2. Agarwal et al. reported higher levels of seminal ORP in infertile patients than in fertile men and proposed an ORP cut-off $\geq 1.36 \mathrm{mV} / 10^{6}$ spermatozoa/ml to discriminate patients based on semen quality (Agarwal, Henkel, et al., 2018; Agarwal, Roychoudhury, Sharma, et al., 2016; Agarwal \& Wang, 2017; Arafa et al., 2018). A multicentre study involving larger cohorts of patients 


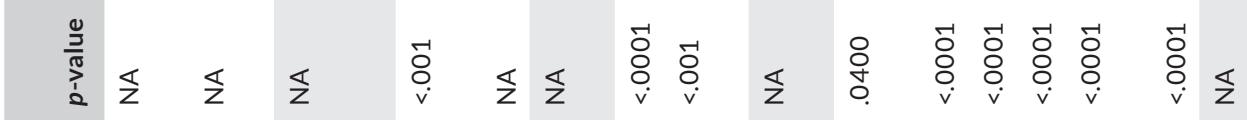

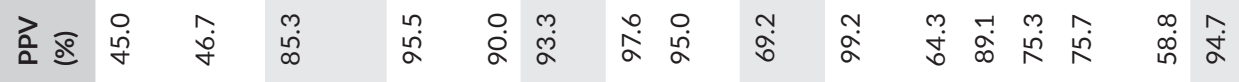

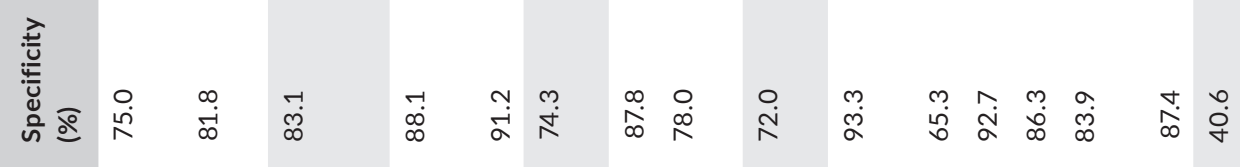

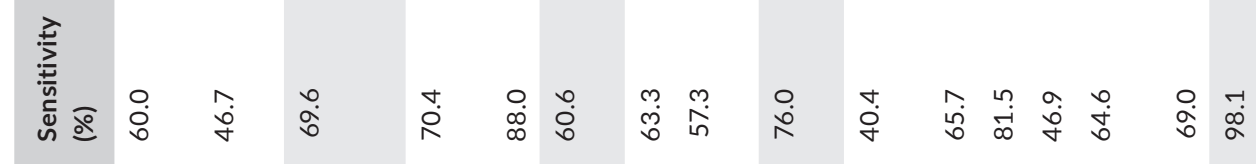

望

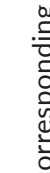

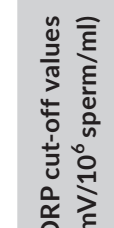

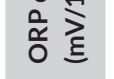

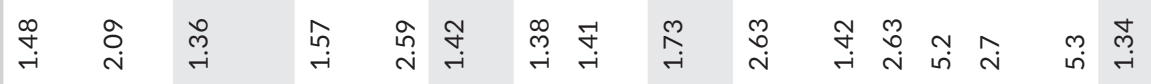

믈

告

要

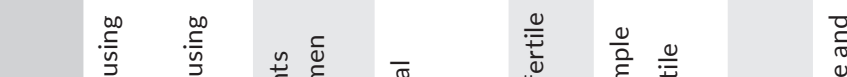

$\frac{\sqrt{\pi}}{\frac{\pi}{\pi}}$

薄

先

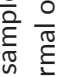

ㄷำ

ป ํำ

पु

苋恋

오몰

인

西

它

은.

흘

咅

인

을

$\frac{\sqrt{2}}{20}$

강

豙

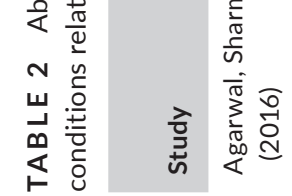

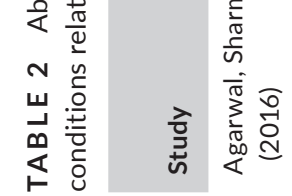

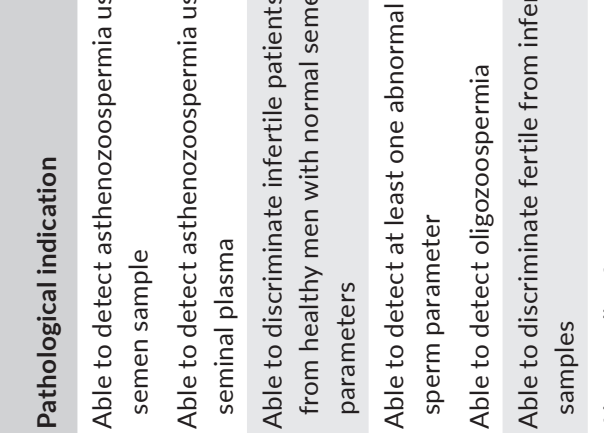

त $\frac{1}{0}$
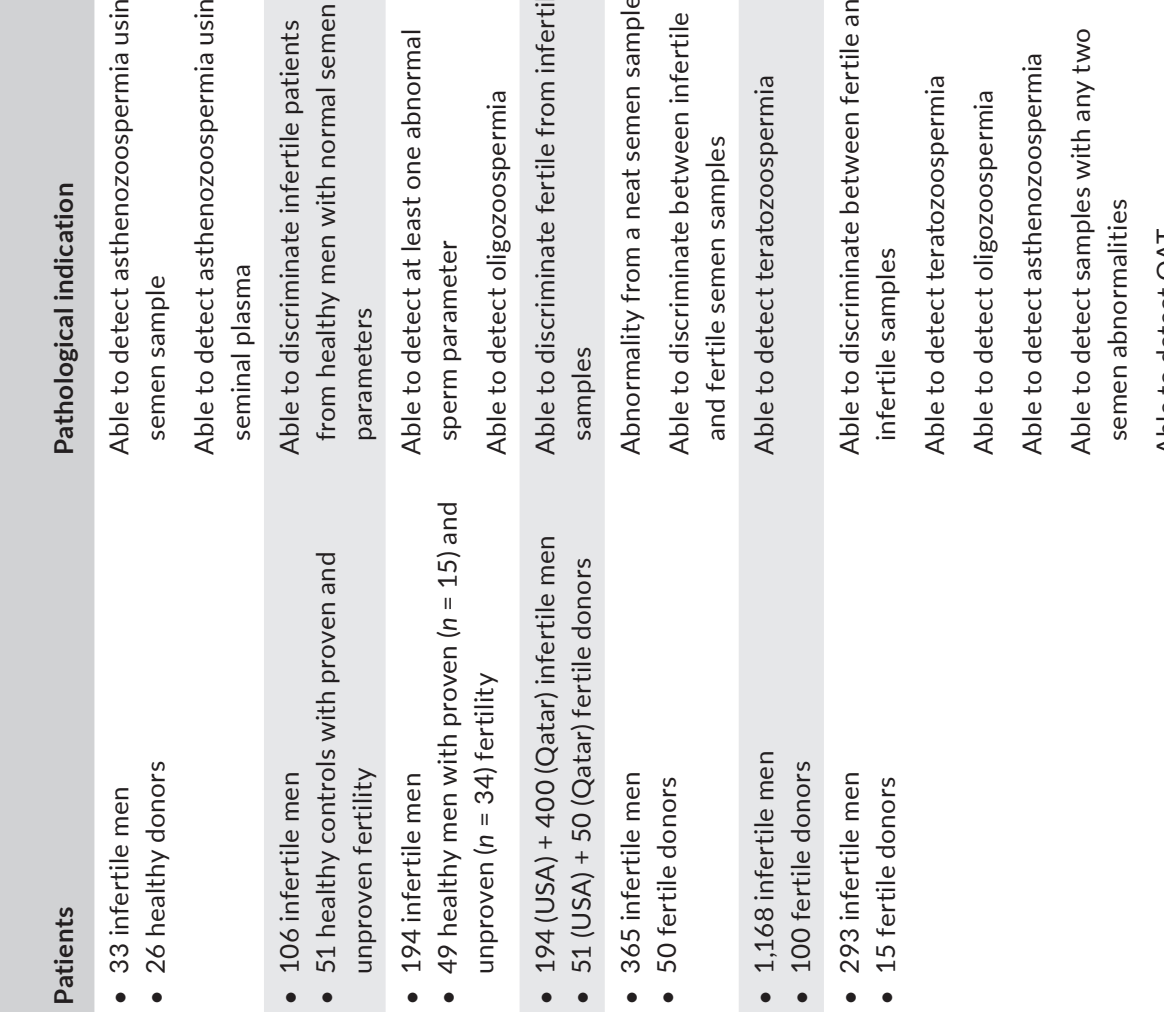

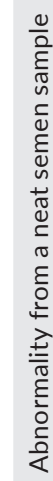
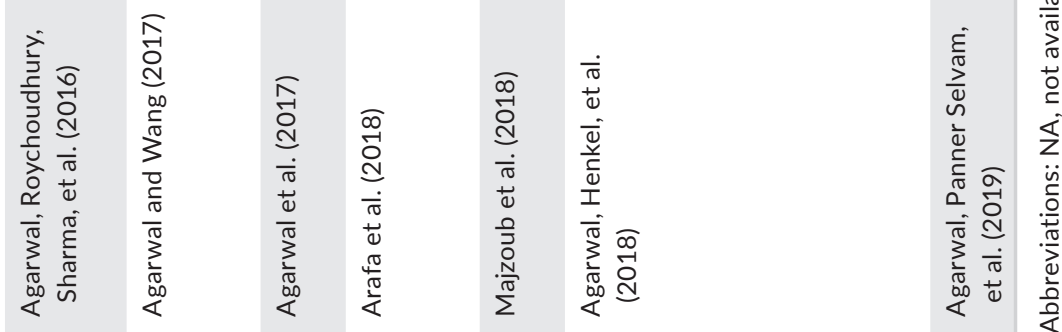


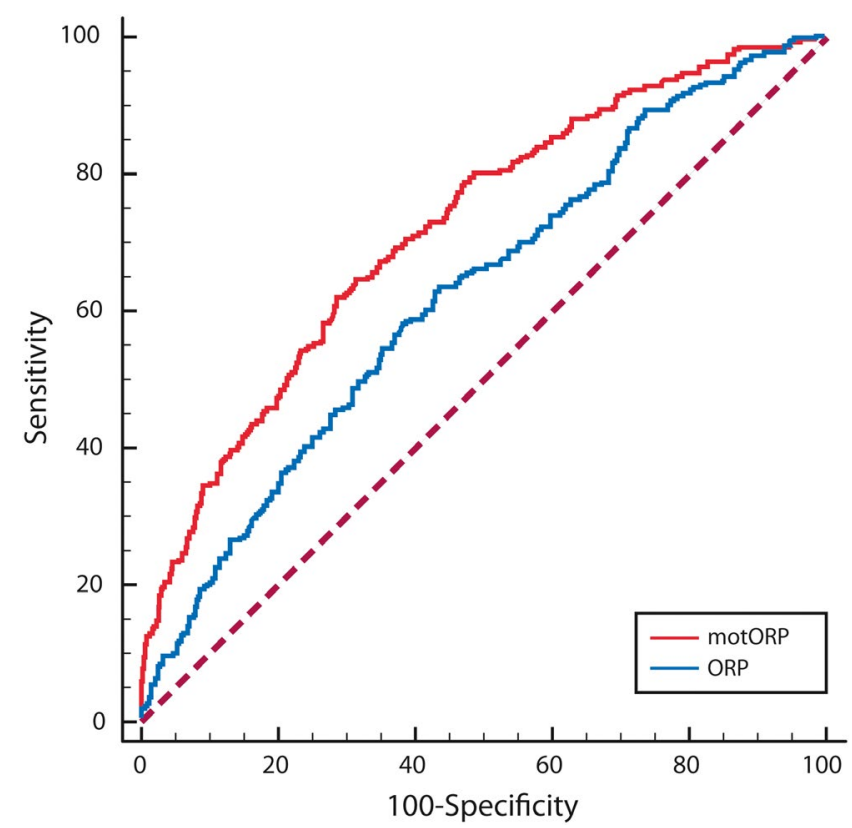

FIGURE 3 Comparison between ROC curves. The ORP (in blue) and motORP (in red) are used to predict the rate of SDF (Elbardisi et al., 2019)

with abnormal semen quality $(n=1,893)$ and normozoospermic men ( $n=199$ ) from nine different institutions around the world suggested a more appropriate cut-off value of $1.34 \mathrm{mV} / 10^{6}$ spermatozoa/ml to discriminate samples based on semen quality (Agarwal, Panner Selvam, et al., 2019). As ORP measurement is a more stable parameter compared to standard semen analysis, ORP is therefore a more reliable parameter in the male infertility workup of patients with different ethnicity (Agarwal, Panner Selvam, et al., 2019).

Seminal ORP was also measured in varicocele patients to discriminate patients based on their fertility status (Arafa et al., 2018). In varicocele patients, oxidative stress plays an important role as an aetiological factor (Agarwal et al., 2012). Varicocele patients with poor semen quality had higher levels of ORP than healthy men of proven or unproven fertility (Agarwal, Roychoudhury, Sharma, et al., 2016; Samanta et al., 2018). A significant negative association has been reported between ORP and testicular volume in varicocele condition ( $r=-.386 ; p<.0001$; Arafa, Henkel, Agarwal, Majzoub, \& Elbardisi, 2019). However, further studies are required to investigate the role of ORP to predict the presence and the severity of varicocele.

As oxidative stress can damage sperm DNA, the association between ORP and sperm DNA fragmentation (SDF) has been investigated. Infertile patients show higher levels of SDF when compared with a fertile control (Santi, Spaggiari, \& Simoni, 2018). SDF and ORP showed a weak positive correlation $(r=.218, p<.0001$; Arafa et al., 2019; Elbardisi et al., 2019; Majzoub et al., 2018) and an ORP value of $1.77 \mathrm{mV} / 10^{6}$ spermatozoa/ml, can be used to predict SDF with moderate sensitivity (63.5\%) and specificity (56.3\%) (Elbardisi et al., 2019). Whereas, the specificity of the assay increased to $71.5 \%$ when the ORP was normalised with the motile sperm fraction
(motORP; Figure 3; Elbardisi et al., 2019). Among all the sperm parameters, total motility is directly affected by oxidative stress. Hence, the use of motORP rather than the ORP normalised against sperm concentration has been suggested to be a better predictor of SDF. However, the predictive power of motORP is still moderate, probably due to the fact that sperm DNA damage can be due to several conditions other than oxidative stress, such as exposure to toxicants, medical therapies as well as defective chromatin compaction and induction of apoptosis (Morris, 2002; Muratori et al., 2015; Sakkas, Seli, Bizzaro, Tarozzi, \& Manicardi, 2003).

ORP is an extracellular marker, while SDF is an intracellular marker of semen quality. Oxidative stress cannot cause immediate damage to the sperm DNA; rather, it is induced due to continuous exposure to ROS over a period of time. This may explain why ORP can hardly predict SDF. Therefore, even if inter-related, SDF and ORP seem to measure independent sperm functions and cannot replace each other in the evaluation of male fertility potential (Elbardisi et al., 2019).

\section{2 | ORP and in vitro fertilization}

Oxidative stress has adverse effects on the in vitro fertilisation process. As such, the redox state of the reagents and culture media used in the assisted reproductive techniques (ART) are critical for the development of embryo. The shifting of the redox balance in medium towards a more oxidative or reductive environment may result in poor reproductive outcomes (Du Plessis, Makker, Desai, \& Agarwal, 2008; Esteves, Roque, \& Agarwal, 2015; Henkel, 2011). In a recent study, the ORP levels were measured by the MiOXSYS device in 10 different culture media used in ART practice to either wash, freeze or culture cells (Panner Selvam, Henkel, Sharma, \& Agarwal, 2018). The authors reported a lower levels of ORP for the sperm cryopreservation and ART culture media analysed in comparison with sperm wash medium; particularly, ORP was lower in a onestep medium than in sequential culture media. This suggests that the one-step medium provides a better capacity to counterbalance oxidative stress, which may be correlated with higher rate of blastocyst formation in one-step medium (Sfontouris et al., 2016). Furthermore, in embryo culture systems, the ORP levels were adjusted using a combination of antioxidants to adjust the ORP levels similar to that of follicular fluid from oocyte donors. Adjustment of ORP levels in culture medium resulted in increased blastocyst formation and ongoing pregnancy rates (Maldonado Rosas et al., 2019).

Similarly, TYB (test yolk buffer) freezing medium showed a lower ORP value due to the presence of antioxidants (Panner Selvam et al., 2018). During cryopreservation, there is an increased production of ROS (Zribi et al., 2010); hence, the use of a low ORP freezing medium may help in maintaining the redox balance. Using a mouse ICSI (intracytoplasmic sperm injection) model, ORP has been used to examine the effect of sperm exposure to polyvinyl pyrrolidone (PVP) and hyaluronic acid (HA) frequently used in ART practice (Roychoudhury et al., 2018). Spermatozoa selected by a 
PVP-containing medium showed a lower ORP value than using a hyaluronic acid-containing medium, which suggests an antioxidant action of PVP. Nakamura et al. (2018) suggested that in vivo intrauterine ORP measurement at cycle days 9-10 could predict pregnancy after frozen-thawed embryo transfer, with significantly lower ORP levels in the pregnant group (Nakamura et al., 2018). A prospective pilot study reported a seminal ORP cut-off equal to $1.36 \mathrm{mV} / 10^{6}$ spermatozoa $/ \mathrm{ml}$ to predict the fertilisation and blastulation rates in a cohort of 50 patients undergoing ICSI (Morris, Siebert, Agarwal, \& Henkel, 2019). However, these studies are still preliminary, and more evidence are needed to assess the role of ORP in evaluating clinical parameters in ART practices.

\section{5 | CONCLUSION}

Seminal ORP represents a valid marker for the evaluation of global redox status. MiOXSYS is a reliable, user-friendly device, with great potential to be used in clinical andrology settings. In association with the standard semen analysis, ORP has already been tested to evaluate semen quality and male fertility status, while further studies are necessary before considering its utility in ART practice.

\section{TAKE-HOME MESSAGE}

- ORP provides an evaluation of the global redox status, unlike other techniques which assess only the oxidants or the antioxidants.

- MiOXSYS is a simple and user-friendly device which can measure ORP in both fresh and frozen samples.

- ORP has been validated in clinical andrology practice as a complementary tool to standard semen analysis for the evaluation of semen quality and male fertility potential.

\section{ORCID}

Manesh Kumar Panner Selvam (iD) https://orcid. org/0000-0002-9120-2278

Renata Finelli iD https://orcid.org/0000-0002-5926-6407

Ashok Agarwal iD https://orcid.org/0000-0003-0585-1026

Ralf Henkel iD https://orcid.org/0000-0003-1128-2982

\section{REFERENCES}

Agarwal, A., Ahmad, G., \& Sharma, R. (2015). Reference values of reactive oxygen species in seminal ejaculates using chemiluminescence assay. Journal of Assisted Reproduction and Genetics, 32(12), 17211729. https://doi.org/10.1007/s10815-015-0584-1

Agarwal, A., Arafa, M., Chandrakumar, R., Majzoub, A., AlSaid, S., \& Elbardisi, H. (2017). A multicenter study to evaluate oxidative stress by oxidation-reduction potential, a reliable and reproducible method. Andrology, 5(5), 939-945. https://doi.org/10.1111/andr.12395

Agarwal, A., Hamada, A., \& Esteves, S. C. (2012). Insight into oxidative stress in varicocele-associated male infertility: Part 1. Nature Reviews Urology, 9, 678-690. https://doi.org/10.1038/nrurol.2012.197

Agarwal, A., Henkel, R., Sharma, R., Tadros, N. N., \& Sabanegh, E. (2018). Determination of seminal oxidation-reduction potential (ORP) as an easy and cost-effective clinical marker of male infertility. Andrologia, 50(3), 1-8. https://doi.org/10.1111/and.12914

Agarwal, A., Panner Selvam, M. K., Arafa, M., Okada, H., Homa, S., Killeen, A., ... Sikka, S. (2019). Multi-center evaluation of oxidation-reduction potential by the MiOXSYS in males with abnormal semen. Asian Journal of Andrology, 21, 1-5. https://doi.org/10.4103/aja.aja_5_19

Agarwal, A., Parekh, N., Panner Selvam, M. K., Henkel, R., Shah, R., Homa, S. T., ... Harlev, A. (2019). Male oxidative stress infertility (MOSI): Proposed terminology and clinical practice guidelines for management of idiopathic male infertility. The World Journal of Men's Health, 37(3), 296-312. https://doi.org/10.5534/wjmh.190055

Agarwal, A., Qiu, E., \& Sharma, R. (2018). Laboratory assessment of oxidative stress in semen. Arab Journal of Urology, 16(1), 77-86. https:// doi.org/10.1016/j.aju.2017.11.008

Agarwal, A., Rana, M., Qiu, E., AlBunni, H., Bui, A., \& Henkel, R. (2018). Role of oxidative stress, infection and inflammation in male infertility. Andrologia, 50(11), e13126.

Agarwal, A., Roychoudhury, S., Bjugstad, K., \& Cho, C. (2016). Oxidationreduction potential of semen: What is its role in the treatment of male infertility? Therapeutic Advances in Urology, 8(5), 302-318. https://doi.org/10.1177/1756287216652779

Agarwal, A., Roychoudhury, S., Sharma, R., Gupta, S., Majzoub, A., \& Sabanegh, E. (2016). Diagnostic application of oxidation-reduction potential assay for measurement of oxidative stress: Clinical utility in male factor infertility. Reproductive BioMedicine Online, 34(1), 48-57. https://doi.org/10.1016/j.rbmo.2016.10.008

Agarwal, A., Sharma, R., Henkel, R., Roychoudhury, S., Sikka, S. C., du Plessis, S., ... Sabanegh, E. (2018). Cumene hydroperoxide induced changes in oxidation-reduction potential in fresh and frozen seminal ejaculates. Andrologia, 50(1), 1-7. https://doi.org/10.1111/and.12796

Agarwal, A., Sharma, R., Roychoudhury, S., Du Plessis, S., \& Sabanegh, E. (2016). MiOXSYS: A novel method of measuring oxidation reduction potential in semen and seminal plasma. Fertility and Sterility, 106(3), 566-573.e10. https://doi.org/10.1016/j.fertnstert.2016.05.013

Agarwal, A., \& Wang, S. (2017). Clinical relevance of oxidation-reduction potential in the evaluation of male infertility. Urology, 104, 84-89. https://doi.org/10.1016/j.urology.2017.02.016

Aitken, R. (2017). Reactive oxygen species as mediators of sperm capacitation and pathological damage. Molecular Reproduction and Development, 84(10), 1039-1052. https://doi.org/10.1002/ mrd. 22871

Aitken, R. J., Baker, M. A., \& O'Bryan, M. (2004). Shedding light on chemiluminescence: The application of chemiluminescence in diagnostic andrology. Journal of Andrology, 25(4), 455-465.

Aitken, R., De luliis, G., Gibb, Z., \& Baker, M. (2012). The simmet lecture: New horizons on an old landscape - oxidative stress, DNA damage and apoptosis in the male germ line. Reproduction in Domestic Animals, 47(SUPPL.4), 7-14.

Arafa, M., Agarwal, A., Al Said, S., Majzoub, A., Sharma, R., Bjugstad, K. B., ... Elbardisi, H. (2018). Semen quality and infertility status can be identified through measures of oxidation-reduction potential. Andrologia, 50(2), 1-6. https://doi.org/10.1111/and.12881

Arafa, M., Henkel, R., Agarwal, A., Majzoub, A., \& Elbardisi, H. (2019). Correlation of oxidation-reduction potential with hormones, semen parameters and testicular volume. Andrologia, 51(5), 1-7. https://doi. org/10.1111/and.13258

Bjugstad, K. B., Rael, L. T., Levy, S., Carrick, M., Mains, C. W., Slone, D. S., \& Bar-Or, D. (2016). Oxidation-reduction potential as a biomarker for severity and acute outcome in traumatic brain injury. Oxidative Medicine and Cellular Longevity, 2016, 1-9. https://doi. org/10.1155/2016/6974257

Cao, T., He, M., Bai, T., \& Liu, H. (2016). Establishment of a method for measuring antioxidant capacity in urine, based on oxidation reduction potential and redox couple $12 / \mathrm{Kl}$. Bioinorganic Chemistry and Applications, 2016, 7054049 
Douglas, C., Parekh, N., Kahn, L., Henkel, R., \& Agarwal, A. (2019). A novel approach to improving the reliability of manual semen analysis: A paradigm shift in the workup of infertile men. The World Journal of Men's Health, 37, e60. https://doi.org/10.5534/wjmh.190088. [Epub ahead of print]

Du Plessis, S. S., Agarwal, A., Halabi, J., \& Tvrda, E. (2015). Contemporary evidence on the physiological role of reactive oxygen species in human sperm function. Journal of Assisted Reproduction and Genetics, 32(4), 509-520. https://doi.org/10.1007/s1081 5-014-0425-7

Du Plessis, S., Makker, K., Desai, N., \& Agarwal, A. (2008). Impact of oxidative stress on IVF. Expert Review of Obstetrics and Gynecology, 3(4), 539-554. https://doi.org/10.1586/17474108.3.4.539

Elbardisi, H., Finelli, R., Agarwal, A., Majzoub, A., Henkel, R., \& Arafa, M. (2019). Predictive value of oxidative stress testing in semen for sperm DNA fragmentation assessed by sperm chromatin dispersion test. Andrology. https://doi.org/10.1111/andr.12743. [Epub ahead of print]

El-Taieb, M. A., Ali, M. A., \& Nada, E. A. (2015). Oxidative stress and acrosomal morphology: A cause of infertility in patients with normal semen parameters. Middle East Fertility Society Journal, 20(2), 79-85. https://doi.org/10.1016/j.mefs.2014.05.003

Esteves, S., Roque, M., \& Agarwal, A. (2015). Outcome of assisted reproductive technology in men with treated and untreated varicocele: Systematic review and meta-analysis. Asian Journal of Andrology, 18(2), 254. https://doi.org/10.4103/1008-682X.163269

Gharagozloo, P., \& Aitken, R. J. (2011). The role of sperm oxidative stress in male infertility and the significance of oral antioxidant therapy. Human Reproduction, 26(7), 1628-1640. https://doi.org/10.1093/ humrep/der132

Henkel, R. (2011). Leukocytes and oxidative stress: Dilemma for sperm function and male fertility. Asian Journal of Andrology, 13(1), 43-52. https://doi.org/10.1038/aja.2010.76

Homa, S., Vassiliou, A., Stone, J., Killeen, A., Dawkins, A., Xie, J., ... Ramsay, J. (2019). A comparison between two assays for measuring seminal oxidative stress and their relationship with sperm DNA fragmentation and semen parameters. Genes, 10(3), 236. https://doi. org/10.3390/genes10030236

Khan, P., Idrees, D., Moxley, M. A., Corbett, J. A., Ahmad, F., von Figura, G., ... Hassan, M. I. (2014). Luminol-based chemiluminescent signals clinical and non-clinical application and future uses. Applied Biochemistry and Biotechnology, 173(2), 333-355. https://doi.org/10.1007/s1201 0-014-0850-1

Mahfouz, R. Z., du Plessis, S. S., Aziz, N., Sharma, R., Sabanegh, E., \& Agarwal, A. (2010). Sperm viability, apoptosis, and intracellular reactive oxygen species levels in human spermatozoa before and after induction of oxidative stress. Fertility and Sterility, 93(3), 814-821. https://doi.org/10.1016/j.fertnstert.2008.10.068

Majzoub, A., Arafa, M., El Ansari, W., Mahdi, M., Agarwal, A., Al-Said, S., \& Elbardisi, H. (2019). Correlation of oxidation reduction potential and total motile sperm count: Its utility in the evaluation of male fertility potential. Asian Journal of Andrology, 21, 1-6.

Majzoub, A., Arafa, M., Mahdi, M., Agarwal, A., Al Said, S., Al-Emadi, I., ... Elbardisi, H. (2018). Oxidation-reduction potential and sperm DNA fragmentation, and their associations with sperm morphological anomalies amongst fertile and infertile men. Arab Journal of Urology, 16(1), 87-95. https://doi.org/10.1016/j.aju.2017.11.014

Maldonado Rosas, I., Agarwal, A., Jimenez Medina, I., Solorzano Vazquez, F., Gabriela Villar, L., Perez Bernal, F. M., \& Panner Selvam, M. K. (2019). The supraphysiological levels of Oxidation Reduction Potential (ORP) present in the human embryo culture media affects blastocysts formation and ongoing pregnancies in IVF cycles. Human Reproduction, 34(Suppl 1), i214.

Marchetti, C. (2002). Study of mitochondrial membrane potential, reactive oxygen species, DNA fragmentation and cell viability by flow cytometry in human sperm. Human Reproduction, 17(5), 1257-1265. https://doi.org/10.1093/humrep/17.5.1257

Morris, A., Siebert, I., Agarwal, A., \& Henkel, R. (2019). Prediction of successful ICSI cycles by Oxidation-reduction potential (ORP) and sperm DNA fragmentation (SDF) analysis. A prospective studies. Human Reproduction, 34(Suppl 1), i8.

Morris, I. D. (2002). Sperm DNA damage and cancer treatment. International Journal of Andrology, 25(5), 255-261.

Muratori, M., Tamburrino, L., Marchiani, S., Cambi, M., Olivito, B., Azzari, C., ... Baldi, E. (2015). Investigation on the origin of sperm DNA fragmentation: Role of apoptosis, immaturity and oxidative stress. Molecular Medicine, 21, 109-122. https://doi.org/10.2119/ molmed.2014.00158

Nakamura, H., Hosono, T., Taniguchi, T., Kumasawa, K., Goa, S., Ono, M., \& Kimura, T. (2018). Prediction of pregnancy after frozen-thawed embryo transfer via in vivo intrauterine oxidation-reduction potential measurements: A pilot study. Reproductive Medicine and Biology, 17(3), 255-261. https://doi.org/10.1002/rmb2.12098

Panner Selvam, M. K., Henkel, R., Sharma, R., \& Agarwal, A. (2018). Calibration of redox potential in sperm wash media and evaluation of oxidation-reduction potential values in various assisted reproductive technology culture media using MiOXSYS system. Andrology, 6(2), 293-300. https://doi.org/10.1111/andr.12461

Pluschkell, S. B., \& Flickinger, M. C. (1995). Improved methods for investigating the external redox potential in hybridoma cell culture. Cytotechnology, 19(1), 11-26. https://doi.org/10.1007/BF00749751

Polson, D., Villalba, N., \& Freeman, K. (2018). Optimization of a diagnostic platform for oxidation-reduction potential (Orp) measurement in human plasma. Redox Report, 23(1), 125-129. https://doi. org/10.1080/13510002.2018.1456000

Roychoudhury, S., Maldonado-Rosas, I., Agarwal, A., Esteves, S. C., Henkel, R., \& Sharma, R. (2018). Human sperm handling in intracytoplasmic sperm injection processes: In vitro studies on mouse oocyte activation, embryo development competence and sperm oxidationreduction potential. Andrologia, 50(4), 1-12. https://doi.org/10.1111/ and.12943

Sakkas, D., Seli, E., Bizzaro, D., Tarozzi, N., \& Manicardi, G. (2003) Abnormal spermatozoa in the ejaculated: Abortive apoptosis and faulty nuclear remodelling during spermatogenesis. Reproductive BioMedicine Online, 7(4), 428-432.

Saleh, R. A., \& Agarwal, A. (2002). Oxidative stress and male infertility: From research bench to clinical practice. Journal of Andrology, 23(6), 737-752.

Samanta, L., Agarwal, A., Swain, N., Sharma, R., Gopalan, B., Esteves, S. C., ... Sabanegh, E. (2018). Proteomic signatures of sperm mitochondria in varicocele: Clinical use as biomarkers of varicocele associated infertility. Journal of Urology, 200(2), 414-422. https://doi. org/10.1016/j.juro.2018.03.009

Santi, D., Spaggiari, G., \& Simoni, M. (2018). Sperm DNA fragmentation index as a promising predictive tool for male infertility diagnosis and treatment management - Meta-analyses. Reproductive BioMedicine Online, 37(3), 315-326. https://doi.org/10.1016/j.rbmo.2018.06.023

Sfontouris, I. A., Martins, W. P., Nastri, C. O., Viana, I. G. R., Navarro, P. A., Raine-Fenning, N., ... Racowsky, C. (2016). Blastocyst culture using single versus sequential media in clinical IVF: A systematic review and meta-analysis of randomized controlled trials. Journal of Assisted Reproduction and Genetics, 33(10), 1261-1272. https://doi. org/10.1007/s10815-016-0774-5

Stagos, D., Goutzourelas, N., Bar-Or, D., Ntontou, A.-M., Bella, E., Becker A. T., ... Kouretas, D. (2015). Application of a new oxidation-reduction potential assessment method in strenuous exercise-induced oxidative stress. Redox Report, 20(4), 154-162. https://doi. org/10.1179/1351000214Y.0000000118

Wang, X., Sharma, R., Gupta, A., George, V., Thomas, A., Falcone, T., \& Agarwal, A. (2003). Alterations in mitochondria membrane potential 
and oxidative stress in infertile men: A prospective observational study. Fertility and Sterility, 80(SUPPL. 2), 844-850.

Wang, X., Sharma, R. K., Sikka, S. C., Thomas, A. J., Falcone, T., \& Agarwal, A. (2003). Oxidative stress is associated with increased apoptosis leading to spermatozoa DNA damage in patients with male factor infertility. Fertility and Sterility, 80(3), 531-535.

WHO (2010). WHO laboratory manual for the examination and processing of human semen (5th ed.). Geneva, Switzerland: WHO.

Zribi, N., Feki Chakroun, N., El Euch, H., Gargouri, J., Bahloul, A., \& Ammar Keskes, L. (2010). Effects of cryopreservation on human sperm deoxyribonucleic acid integrity. Fertility and Sterility, 93(1), 159-166.
How to cite this article: Panner Selvam MK, Finelli R, Agarwal A, Henkel R. Evaluation of seminal oxidationreduction potential in male infertility. Andrologia.

2021;53:e13610. https://doi.org/10.1111/and.13610 